\title{
Prototype formation in very short-term memory
}

\author{
ROBERT L. SOLSO, MATHEW HECK, and CURT MEARNS \\ University of Nevada, Reno, Nevada
}

\begin{abstract}
Following high-speed presentation of stimuli, subjects falsely recognized prototype numbers and words as being among the set of original stimuli. The results suggest high-speed abstraction of information by subjects during or shortly after high-speed perception. This abstraction of a class of information may leave a memory trace that is a more memorable representation of events (as measured by recognition techniques) than are the perceived stimuli. These results suggest that very short-term processing of information is more complicated than simple sequential dualistic models of memory assume.
\end{abstract}

The present research deals with the cognitive properties that take place during the memory stage commonly known as short-term memory. Of particular interest are the processes that take place during the very early stages following presentation of stimuli or during very short-term memory.

Although the distinctions between short-term memory and long-term memory seem to be waning (i.e., information in short-term memory seems to be coded in ways once ascribed only to long-term memory-e.g., a visual code [Posner \& Keele, 1967], or a semantic code [Wickens, 1972]), many researchers have continued to embrace a dichotomous memory system because it provides a "logical" framework for the representation of memory. In addition, a dual memory system is a meaningful heuristic that facilitates communication between scientists and students because it is easy to grasp and explain. It also provides a useful organizational scheme upon which new theories and research can be built. And finally, although less than perfect, it provides a better structure for the conceptualization of memory than do most other models.

In spite of such support for a dualistic concept of memory, a number of findings and theories have questioned its validity. A basic premise of the information processing paradigm is that as information is processed at different stages, unique operations take place at each stage. One distinction between long-term and short-term memory is that abstract representations are the exclusive domain of long-term memory.

The subjects of abstract memories, prototype formation, and category learning occupy a central position in psychology. These topics have been approached from three general models: (1) an exemplar model, which holds that subjects store exemplars of a category and then make judgments of new instances according to their relative similarity to the stored exemplars (e.g., Medin \& Schaffer, 1978); (2) a feature-frequency model, which holds that

Portions of this article are based on a paper presented at the annual meeting of the Psychonomic Society, November 6, 1987, Seattle. Correspondence should be addressed to R. L. Solso, Department of Psychology, University of Nevada, Reno, NV 89557-0062. subjects store the frequencies of occurrence of stimuli and then classify new patterns according to the likelihood that those patterns arise from each of the categories (Franks \& Bransford, 1971; Solso \& Raynis, 1979); and (3) a prototype model, which suggests that subjects form a central tendency or modal representation of categories and then classify new instances according to central prototypes (Homa, 1984).

It has been suggested (Solso \& Raynis, 1979) that subjects may use different strategies in forming abstract memories, depending on the types of information presented. Thus, stimuli rich in features, such as numbers or geometric figures, may favor the storage of information according to a feature-frequency model, whereas other stimuli that encourage semantic abstraction, and that are somewhat devoid of features, may favor exemplar or prototype models.

Several experiments have been done on the issue of semantic organization and high-speed scanning (see Burrows \& Okada, 1973; Jones \& Anderson, 1982; and Okada \& Burrows, 1973). Although these studies have focused on the scanning process in short-term memory, they have, incidentally, provided data that demonstrate semantic organization of material held in an immediate memory store.

In the present experiments, we examined the notion of abstract representations-namely, prototype formation-in very short-term memory by using the Sternberg paradigm with two classes of material (numbers and words); we found that subjects tended to false alarm on both numbers and semantic information, which suggests that some abstraction process occurs in very short-term memory.

\section{METHOD}

\begin{abstract}
Materials
The method and composition of items were similar to that of Solso and McCarthy (1981). A prototype number was created, which in one condition was composed of a two-digit number and in another condition a three-digit number. These numbers were selected with the following constraints: no single digits were repeated within the prototype number (e.g., 44, 733), and meaningful numbers were excluded (e.g., $50,123)$. From these two- or three-digit prototype numbers, two- and three-digit series of exemplar numbers were generated. In the two-digit
\end{abstract}


group, two sets of exemplars were produced-one set that had four exemplars and another that had six exemplars. In the three-digit group, six and nine three-digit numbers were created. These exemplar numbers followed the same constraints mentioned above. Exemplar numbers shared common positional features with the prototype numbers. In the case of two-digit series, two of the first positions and two of the second numbers (four-exemplar set) were identical to the prototype. In the case of the three-digit series, three of the first and three of the second numbers (six-exemplar set) corresponded to the prototype number. In the second set of digits in which three numbers were used, the exemplar set contained shared positional features of the prototype number, which was repeated either two times in each of the three positions for the six-number series, or three times in each of the three positions for the nine-number series. In no case did two of the numbers in the prototype appear in the same position in any exemplar set. An example of the composition of the lists follows: In a two-digit set, the prototype number could be 83 , and the exemplars, $89,82,43$, and 73 . For a threedigit set, the prototype could be 692 , and examples of the exemplars, 673,497 , and 852 , in which each of the digits in the prototype is repeated in its respective position, but no more than a single prototype number is found in any exemplar.

In the word condition, lists of four words were used. These words were synonyms of a prototype word (the use of prototype here is meant to imply "of the same class," not the epitome of the class). In total, four different types of lists were used. In one condition, four words were used, only one of which was a synonym of the prototype word. Three other conditions were formed, in which two, three, or four of the exemplar words were synonyms of the prototype word. The words in the exemplar sets and recognition sets were matched for word frequency, and no words of less than 10 per million were used. An example of an exemplar set is "LARGE, BIG, GRAND, HUGE." In this case the probe words might be "VAST, GRAND, and HORSE," in which the prototype item, an old item, and a new item are listed, respectively.

\section{Design}

A standard Sternberg paradigm was used. The design was a repeated measures experiment in which digits or words were both presented randomly for each subject. Order was counterbalanced. Subjects received eight warm-up trials for the number condition and six warm-up trials for the word condition. Additional warm-up trials were administered

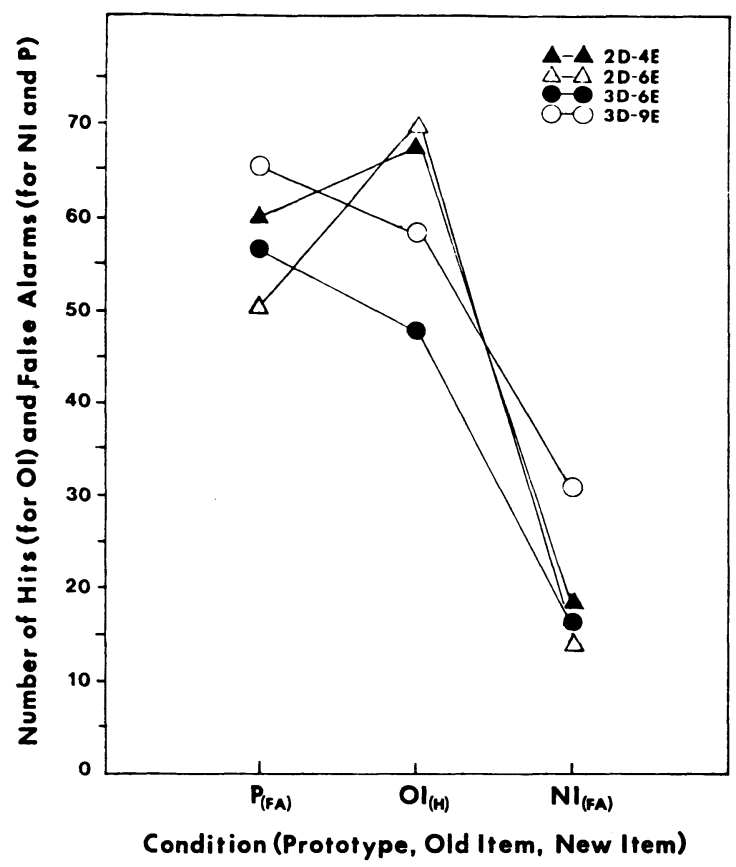

Figure 1. Hits and false alarms for prototype, old items, and new items.

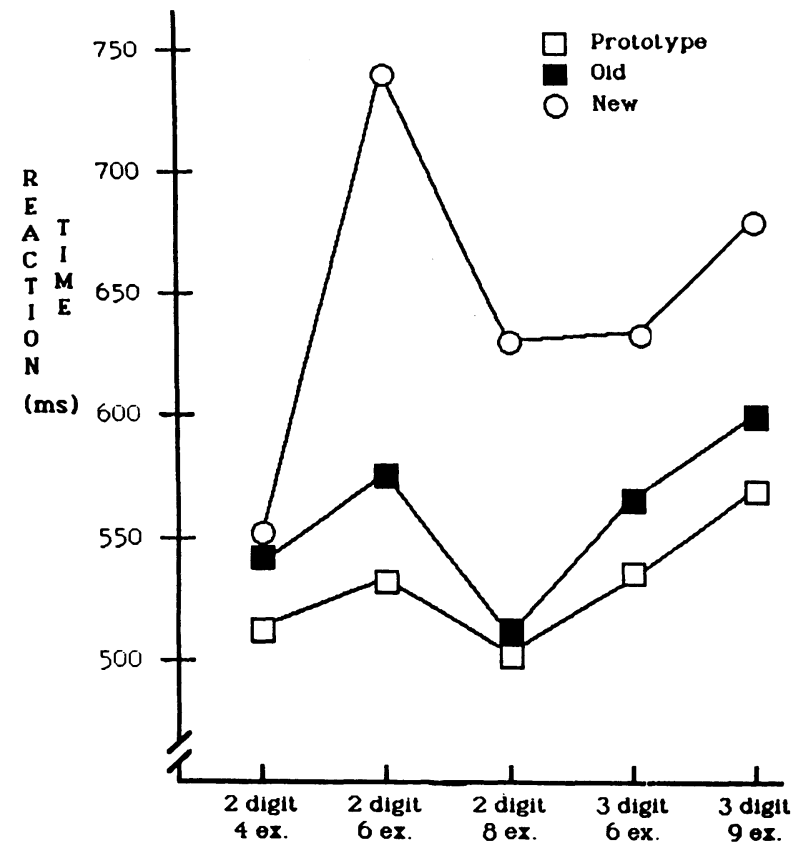

Figure 2. Reaction times by conditions for prototypes (false alarms), old items (hits), and new items (false alarms).

if needed. In the digit condition, the series of two- or three-digit numbers were displayed on an IBM PC at the rate of 1 item per $1.2 \mathrm{sec}$. The same conditions applied to the word condition. A 2-sec interval was used between the presentation of the exemplars and the test condition. The test phase had three digits or words, depending on the condition. In the digit condition, a new digit, an old digit, and the prototype digit were presented randomly. In the word condition, subjects were presented with an old word, a new word, and a synonym of an old word or several words. Following the presentation of the exemplar items, subjects were instructed to identify as fast as possible whether or not the item presented in the second series was an old item (previously in the set) or a new item (previously not in the set) by pressing one of two keys that had been marked.

\section{Subjects}

Twenty-eight students enrolled in an upper division/graduate level course in cognitive psychology, who received class credit for their participation, served as the subjects.

\section{RESULTS}

Two indices of performance were used: Response decision and reaction time. Responses were classified as (1) hitsold items, (2) misses-old items, (3) false alarms-prototype, (4) false alarms-new items, (5) correct rejections-prototype, and (6) correct rejections-new items.

In the first condition, with numbers in all five cells, subjects overwhelmingly gave false alarms to the prototype number as opposed to other new numbers (see Figure 1). The overall ratio of false alarms for prototype numbers was three times greater than the ratio for other new numbers. Evidence for pseudomemory was also found in the conditions in which the information load was large-for example, in the instances of two digits-eight exemplars, three digits-six exemplars, and three digitsnine exemplars. As can be seen in Figure 2, reaction time 


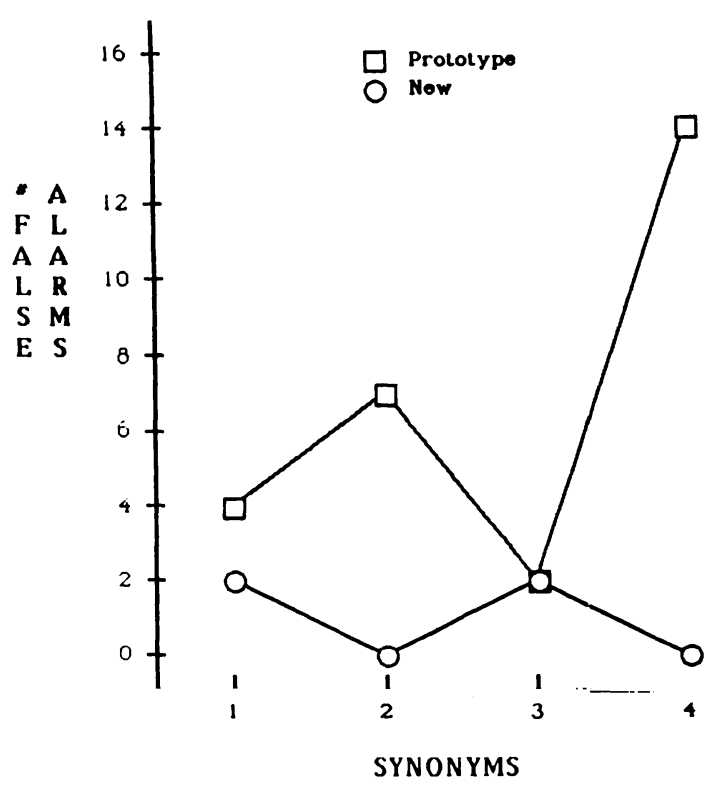

Figure 3. Number of false alarms for prototype words and new words by number of synonyms in the original set.

data indicated that in each of the five cells the reaction time for prototype numbers was faster than that for comparable hits (i.e., judging an old item to be an old item) and each of the five cells of the correct rejection (i.e., correctly identifying a new item [with the exception of the prototype number] as a new item) required more time than did comparable hits.

Data from the word condition indicated some semantic processing in very short-term memory. Subjects tended to give false alarms on test words that were semantically related to exemplar words. As anticipated, the number of false alarms increased as the number of semantically related words in the initial set increased. The overall rate of false alarms for semantically related words was about six times greater than the false alarms for unrelated words (see Figure 3).

\section{DISCUSSION}

In both experiments, the total time used for presentation of items to be memorized ranged from 4.8 to $10.8 \mathrm{sec}(6-12 \mathrm{sec}$, including the time between the presentation of memory set items and the recall stage). These time parameters are well within the limits of what is commonly called short-term memory. Our results show clearly that abstraction of both numerical and semantic information takes place in very short-term memory. In the case of numerical abstraction, the data indicate that in conditions in which the information load may have exceeded immediate memory span, subjects identified prototype numbers as being previously seen items more frequently than they identified items previously presented, even if the items had been seen within about $6 \mathrm{sec}$ of the decision. The tendency to form a more representational memory trace of abstracted stimuli as opposed to actually perceived stimuli has been called pseudomemory. In the case of the numerical stimuli, evidence is presented here, for the first time, of pseudomemory in short-term memory.

In the instance of word stimuli, pseudomemory was neither found nor expected. The data did, however, indicate that within the very early processing of words, semantic processing occurs. The degree of generalization of semantic information appears to be in direct proportion to the number of semantically related exemplars: in the case of a single exemplar, no false alarms on target synonyms were found. The false alarm data are corroborated by the reaction time data, which show a slight hesitancy on the part of subjects when confronted with a word on the test stage that was categorically similar to a word or words presented in the initial stage.

These findings have troubling implications for dualistic memory theories within the context of an information processing model. If the model, as originally proposed, is characterized by the occurrence of discreet functions associated with each of the hypothetical stages through which information is processed, and if abstraction of information is thought to be a function of long-term memory, then our data, which show clearly that the abstraction of both numbers and words occurs in short-term memory, raise serious questions about the differentiation of two stages of memory on the basis of information abstraction.

At the very least, it would appear that the traditional model of dualistic memory needs to be revised. An alternative model would be one in which the abstraction of information plays a cardinal role in memory models. In a revised model of memory, we suggest that the distinction between short-term and long-term memory may still have some utility (especially as descriptive terms that are tied to the time sequence through which information is processed); however, a pervasive characteristic of memory is the abstraction of sensory information, which appears to begin very early in the processing of human memory. If one subscribes to a limited-capacity model of human information processing, it may be critical for parsimonious coding of information in long-term memory to begin the process of abstraction soon after information is perceived, especially if the amount of information exceeds immediate memory capacity.

Finally, with regard to the way in which information is processed in short-term memory, it would appear that although the Sternberg paradigm is still a very useful technique for the study of short-term memory, the theoretical model originally proposed by Sternberg (1966), in which information is accessed by means of an exhaustive and non-selfterminating search mechanism, seems inconsistent with our results, in which prototype numbers are more accessible than some perceived numbers. In a later paper, Sternberg (1969) does provide a mechanism for accessing information that exceeds the capacity of active memory, in which subjects may use some organizational cue to reduce the items to be scanned. Perhaps the current results can be interpreted within the context of organization cues.

In addition, the widely held metaphor of short-term memory as consisting of a series of slots in which well-defined information is temporarily stored as it is perceived is inconsistent with the present findings. We suggest a model of storage in which environmental stimuli are subjected to a form of generalization in which immediately perceived events are coded in relationship to other immediate events. The combination of these events is hypothesized to be summed algebraically, so that the most frequently experienced feature forms a memory representation that can be more available for recognition than an actually perceived event. The data presented here support such a notion. In the case of numbers, not only did subjects falsely recognize prototype numbers more frequently than exemplar numbers but they also responded affirmatively more rapidly than they did for exemplar numbers, which we interpret as evidence for the greater representational value of prototype numbers in short-term memory.

Specifically, consider the case of the condition in which four twodigit numbers were presented at the rate of $1.2 \mathrm{sec}$ per item. If the set included the numbers $68,43,73$, and 67 , the prototype number would be 63 . We suggest that each digit in the two-digit numbers, as well as the two-digit number itself, is immediately subjected to a generalization effect that leaves a memory trace. The most frequently experienced numbers (individual digits and two-digit numbers) are combined with other numbers in the subject's present memory store. In the case indicated, the repetition of the digit 6 in the first position and 3 in the second position would tend to be combined representationally in such a way that upon a recognition trial the subject falsely recognizes the com- 
bined form as a positive instance of his/her immediate memory store. Semantically related items also seem to be coded in a generalized sense, but these effects are less robust in the present research paradigm.

\section{REFERENCES}

Burrows, D., \& OKADA, R. (1973). Parallel scanning of semantic and formal information. Journal of Experimental Psychology, 97, 254-257.

Franks, J. J., \& Bransford, J. D. (1971). Abstraction of visual patterns. Journal of Experimental Psychology, 90, 65-74.

Homa, D. (1984). On the nature of categories. In G. H. Bower (Ed.), The psychology of learning and motivation: Advances in research and theory (Vol. 18, pp. 49-94). New York: Academic Press.

JONES, W. P., \& ANDERSON, J. R. (1982). Semantic categorization and high-speed scanning. Journal of Experimental Psychology: Learning, Memory, \& Cognition, 8, 237-242.

Medin, D. L., \& SCHAFFER, M. M. (1978). A contextual theory of classification learning. Psychological Review, 85, 207-238.

OKADA, R., \& BurRows, D. (1973). Organizational factors in highspeed scanning. Journal of Experimental Psychology, 101, 77-81.
Posner, M. I., \& KeEle, S. W. (1967). On the genesis of abstract ideas. Journal of Experimental Psychology, 77, 353-363.

Solso, R. L., \& McCarthy, J. E. (1981). Prototype formation: Central tendency model vs. attribute-frequency model. Bulletin of the Psychonomic Society, 17, 10-11.

Solso, R. L., \& RAYNIS, S. A. (1979). Prototype formation from imaged, kinesthetically, and visually presented geometric figures. Journal of Experimental Psychology: Human Perception \& Performance, 5, 701-712.

STERNBERG, S. (1966). High-speed scanning in human memory. Science, 153, 652-654.

STERnBerg, S. (1969). Memory scanning: Mental processes revealed by reaction-time experiments. American Scientist, 57, 421-457.

WICKENS, D. D. (1972). Characteristics of word encoding. In A. Melton \& E. Martin (Eds.), Coding processes in human memory (pp. 191215). Washington, DC: Winston.

(Manuscript received November 23, 1992.) 